\title{
Correlations between acute atrial fibrillation and local earth magnetic field strength
}

\author{
Greta Žiubryte் $^{1}$, Gediminas Jaruševičius ${ }^{2}$, Jorūnė Jurjonaitė ${ }^{3}$, Mantas Landauskas ${ }^{4}$, \\ Rollin McCraty ${ }^{5}$, Alfonsas Vainoras ${ }^{6}$ \\ ${ }^{1}$ Faculty of Medicine, Academy of Medicine, Lithuanian University of Health Sciences, \\ Kaunas, 44307, Lithuania \\ ${ }^{2}$ Department of Cardiology, Hospital of Lithuanian University of Health Sciences Kaunas Clinics, \\ Kaunas, 50161, Lithuania \\ ${ }^{2,6}$ Cardiology Institute, Lithuanian University of Health Sciences, Kaunas, 44307, Lithuania \\ ${ }^{3}$ Faculty of Medicine, Academy of Medicine, Lithuanian University of Health Sciences, \\ Kaunas, 44307, Lithuania \\ ${ }^{4}$ Department of Mathematical Modelling, Kaunas University of Technology, Kaunas, Lithuania \\ ${ }^{5}$ HeartMath Institute, California, USA \\ ${ }^{6}$ Sport Institute, Lithuanian University of Health Sciences, Kaunas, 47181, Lithuania \\ ${ }^{1}$ Corresponding author \\ E-mail: '19reta.ziubryte@gmail.com, ${ }^{2}$ gedijaru@yahoo.com,3jjjorune@gmail.com, \\ 4mantas.landauskas@ktu.lt, ${ }^{5}$ rollin@heartmath.org, ${ }^{6}$ alfavain@gmail.com
}

Received 2 November 2018; received in revised form 19 December 2018; accepted 27 December 2018 DOI https://doi.org/10.21595/chs.2018.20430

Check for updates

Copyright (C) 2018 Greta Žiubryte, et al. This is an open access article distributed under the Creative Commons Attribution License, which permits unrestricted use, distribution, and reproduction in any medium, provided the original work is properly cited.

\begin{abstract}
Objectives: Atrial fibrillation is the most frequent cardiac arrhythmia affecting over 3 percent and appears to be increasing in general population. In addition to widely discussed such risk factor as obesity, arterial hypertension, electrolytes disbalances and dysfunction of thyroid, there is more and more evidence of human heath interactions with environment parameters such as humidity, temperature, the lunar and the solar activity. Atrial fibrillation, a disorder of heart conductive system, in several studies have been indicated as affected by local Earth magnetic field changes. The study was aimed to analyse possible correlations between the power in the local Earth time-varying magnetic field and admission due to atrial fibrillation. Methods: Two-hundred-fifty-one patients diagnosed with acute atrial fibrillation and treated in Department of Cardiology of Hospital of Lithuanian University of Health Sciences Kaunas Clinics during year of 2016 were retrospectively included into the study. Weekly prevalence of acute atrial fibrillation was compared with weekly summarised changes in the local Earth magnetic field strength. One-year was divided into two time periods according to week number: the first period included weeks from 1 to 26 and the second period included weeks from 27 to 52. Results: Analyses have shown from weak to moderate significant correlations. Tendencies towards higher power magnetic field in low frequently ranges to be associated with higher admission rates were noticeable throughout all analysed periods. Atrial fibrillation concomitant with arterial hypertension was indicated as combination increasing severity of correlation coefficient. Conclusion: Significant correlations between acute atrial fibrillation and the local Earth time varying magnetic field changes were found. Increased magnetic field in low frequency ranges are associated with episodes of acute atrial fibrillation. Arterial hypertension is significantly associated with higher admission due to atrial fibrillation rate under low frequency local Earth magnetic field range.
\end{abstract}

Keywords: atrial fibrillation, geomagnetic field, Earth's magnetic field, magnetic field, cardiology, arrhythmias, cardiac arrhythmia.

\section{Introduction}

Cardiac arrhythmias are widely discussed, however, there are still more questions than answers, so they are important clinical condition for deep investigations. Atrial fibrillation (AFib) is the 
most frequent cardiac arrhythmia affecting over 3 percent of general population, the number of which increases with age from less than $1 \%$ in young adults to almost $10 \%$ in people older than 80 years [1-3]. They are also associated with causing severe complications such as heart failure and ischemic stroke and severely worsening the quality of life [3-6]. It is known that intracellular junctions' impairment in the heart conductive system, most frequently a consequence of atriums' enlargement, may manifest as AFib. An abnormal electrical substrate responsible for AFib is located around intrinsic ganglia which is one of the most important components in cardiac nervous system [7]. As heart is abundantly supplied by nervous fibers, it is one of the most sensitive organs to the local time-varying magnetic field (TVMF) changes [7]. In addition, it may occur due to serum and intracellular electrolytes disbalance interfering myocardial electric conductivity [8]. Significant association with AFib and TVMF changes has been shown in recently discussed study [9]. Even more, AFib onsets association with thyroid dysfunction has been noticed in clinical practice. Not surprisingly, AFib dependency with thyroid dysfunction has been proved by Selmer et al., [10]. Human hormonal variability in accordance to changes in TVMF has been reported in recent study [11]. Even more, it was stated that hormonal activity diverges in the different halves of the year. It is interesting that in the first half of the year when the TVMF is weaker, thyroid produces more hormones and vice versa in the second half of the year [11]. Nevertheless, in some cases the cause of AFib remains unclear as it occurs in patients with normal size of heart chambers, normal thyroid function and with any or minor serum electrolytes disbalances.

There is strong evidence of extracted animal cells' sensitivity to changes in TVMF frequency and strength [11-14]. Even more, TVMF regulatory effect, which is expected to be related to ion cyclotron resonance mechanism $[11,13]$ have been proven on extracted myocardial cells under laboratory conditions $[11,15]$. Accurate prevalence of human sensitivity to TVMF changes is unknown, but it is possible that one of five - ten people in general population may be sensitive to TVMF fluctuations and develop various signs and symptoms [16]. This was discussed in World Health Organisation (WHO) meeting purposed for International Workshop on Electromagnetic Field Hypersensitivity conducted in 2004, in Prague, Czech Republic [16].

Several studies have shown significant TVMF changes impact on increased hospital admission due to exacerbation of chronic disease $[17,18]$. Palmer et al. reported that approximately $75 \%$ of geomagnetic (GM) storms are followed by increased hospitalization due to cardiological and neurological diseases [18]. Reliable associations between TVMF changes and variability of heart rate (HR), blood pressure (BP) and neural regulation in heart autonomic nervous system have been published in several studies [19]. Even more, strong correlations between GM activity and onsets of cardiac emergencies including acute myocardial infarction (AMI) and crisis of arterial hypertension $(\mathrm{AH})$ have been published [12, 17, 20-22].

TVMF is constantly changing with its lowest point in winter and slowly increase since spring till it reaches the peak in the middle-end of summer [11]. TVMF strength abruptly decreases in the autumn and reaches its lowest point at the middle of winter [11]. These dynamics are given in Fig. 1.

TVMF in different frequency intervals has different effects on humans. It is proposed that low frequencies have positive, while high frequencies have negative effects on human regulatory systems [11]. Despite that, both low and high frequency intervals might be associated with both positive and negative effect on human health [23-25]. Interactions between human beings and magnetosphere parameters might be illustrated by the overlap between Schumann resonance frequencies and human brain activity registered on electroencephalogram (EEG) (Table 1)

Table 1. Overlap between Schumann resonance frequencies and electroencephalogram ranges [11]

\begin{tabular}{|c|c|c|c|c|c|c|}
\hline Schumann resonances & SDelta & STheta & SAlpha & SBeta & SGamma & Whole interval range \\
\hline EEG ranges, Hz & $0-3.5$ & $3.5-7$ & $7-15$ & $15-32$ & $32-65$ & $0-65$ \\
\hline
\end{tabular}

Our interest in cardiac arrhythmias and sparse evidence of TVMF changes influence on AFib onsets encouraged us to perform this study, the purpose of which was to analyse correlations 
between TVMF changes and hospital admissions due to AFib and define possible mechanisms of pathogenesis. To our knowledge this is the first study analysing TVMF influence on AFib onsets in quite big study sample.

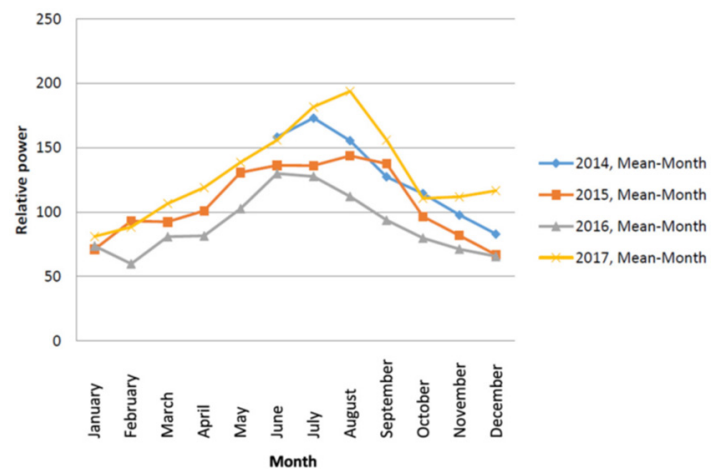

Fig. 1. The changes of strength of TVMF in Lithuania between 2014 June and 2017 December $[11,12]$

\section{Methods}

This is a continued study of our previously published research on acute coronary syndrome patients [11]. In previous study described methods were applied for collecting study population, chosen study and analyses intervals, magnetometer data, statistical analyses and spectral analysis of the magnetometer data where the same as in our earlier publication [11]. This study is focused on relationship between acute atrial fibrillation and TVMF changes.

\subsection{Study population}

Two-hundred-fifty-one (142 (56.6\%) male and 109 (43.4\%) female) patients admitted to the Cardiology Department of Hospital of Lithuanian University of Health Sciences Kaunas Clinics due to acute atrial fibrillation (AAFib) within the year of 2016 were retrospectively included in the study. The mean age of males and females was $66.61( \pm 8.02)$ and $68.63( \pm 7.63)$, respectively. In total, 221 of the $251(88.05 \%)$ patients $(123(86.62 \%)$ male and $98(89.91 \%)$ female) had a history of AH and 91 (36.25\%) of the patients were obese. There were no significant differences between the male and female groups. Due to the relatively low number of patients, we did not divide it into groups by age for the current analyses. Gender related weekly prevalence of AAFib is shown in Fig. 2. Patients' baseline characteristics were collected from medical records, after patients' written consent was obtained. Local Ethical committee approval was given [11].

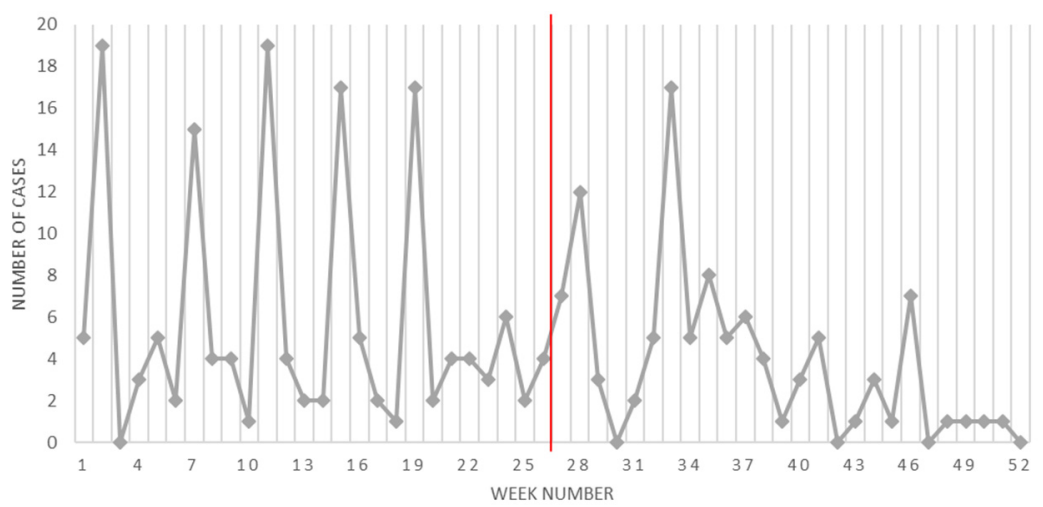

Fig. 2. Weekly prevalence of AAFib. Two periods of the year is separated by the red line 


\subsection{Study and analyses time intervals}

The one-year was divided into two time periods according to week number: the first period included weeks from 1 to 26 and the second period included weeks from 27 to 52 . We divided the time period due to difference in TVMF activity and the human thyroid and adrenal cortex hormone regulation in the first and the second halves of the year [11].

\subsection{Inclusion and exclusion criteria}

All patients admitted to Department of Cardiology in 2016 due to AAFib revealed by electrocardiogram (ECG) which persists up to 48 hours and with clear onset time were included into the study. For all patients, 12-leads ECG was acquired. Patients with unclear time of AFib onset and with exacerbated symptoms of permanent AFib were excluded.

\subsection{Magnetometer data}

The TVMF intensity was measured by the Global Coherence Monitoring Network magnetometer located In Lithuania. For current analyses data observed in east/west direction was used, because there is evidence that this direction is more significantly correlated with human health parameters. Magnetometers signals were digitalized with 24-bit data acquisition system (Symmetric Research, Las Vegas, NV, USA) at a rate of $130 \mathrm{~Hz}$ and uploaded hourly to a cloud data storage site for offline processing. Hourly data was downloaded to a personal computer and transformed into successive thirty-second length segments for each of whom the power spectral density (PDS) was calculated, which were daily and weekly. The sum of them in $0-65 \mathrm{~Hz}$ frequency range was calculated over the study period. TVMF was analysed in five frequency ranges overlapping with the EEG ranges $[\mathrm{Hz}]$. To distinguish those frequency ranges in EEG from the magnetic field in same range as the Schumann resonances occur, we add an S in front: (SDelta) [0; 3.5], (STheta) [3.5; 7], (SAlpha) [7; 15], (SBeta) [15; 32], (SGamma) [32; 65]. it was added full frequency interval range $[0 ; 65]$ in which all intervals were summed up all power in one to observe correlations in summarised TVMF spectrum [11].

For investigation of how power of Earth TVMF influence number of AFib power in every frequency interval was evaluated in 5 levels ( 1 - lowest, 5 strongest in every frequency interval). Step between levels was calculated as $(\max -\min ) / 5$.

\subsection{Statistical analysis}

The statistical analysis was performed using the software package SPSS 20.0 and MS Office Excel 2014. Nonparametric Mann-Whitney U test was used for the comparison of two independent samples. Pearson correlation coefficient for the linear correlation between two variables was calculated. The level of $p<0.05$ was considered statistically significant [11].

\subsection{Mathematical approach to computing powers of magnetic field from the magnetometer data}

For spectral analysis of the magnetometer data short time Fourier transform (STFT) was used. These methods are explained more detailed in our previous publication by Žiubrytè et al., [11] which is a part of our project of Environment parameters influence on heart disease [11].

\section{Results}

Significant correlations between weekly admission due to AAFib and average weekly TVMF strength in five frequency ranges were found. Analyses of the whole year model have not shown any significant correlations in both groups (Fig. 3). But tendencies of stronger correlations in high 
frequency ranges in males and in low frequency ranges in females were observed.

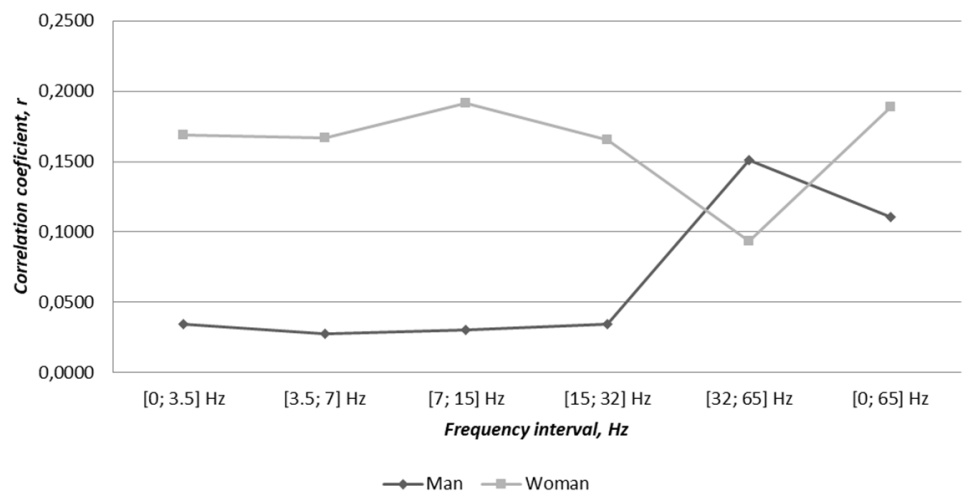

Fig. 3. Correlations between gender related cases of atrial fibrillation and TVMF changes through 2016

After dividing the year into two time periods, positive correlation coefficients were found in both groups through the first half of the year (Fig. 4), with the exception of SGamma range in both groups which was the only negative correlation. SDelta $[0 ; 3.5] \mathrm{Hz}$ frequency range significantly correlated with higher occurrence of AAFib in males and females through the first half of the year ( $r=0.384, p=0.045$ and $r=0.454, p=0.020$, respectively). In addition to the SDelta range, in the female group, STheta $[3.5 ; 7] \mathrm{Hz}$ range showed a moderate but significant correlation coefficient, $r=0.405, p=0.040$. The results suggest, that higher TVMF activity in low frequency ranges is associated with provoking the onset of AFib independently of gender.

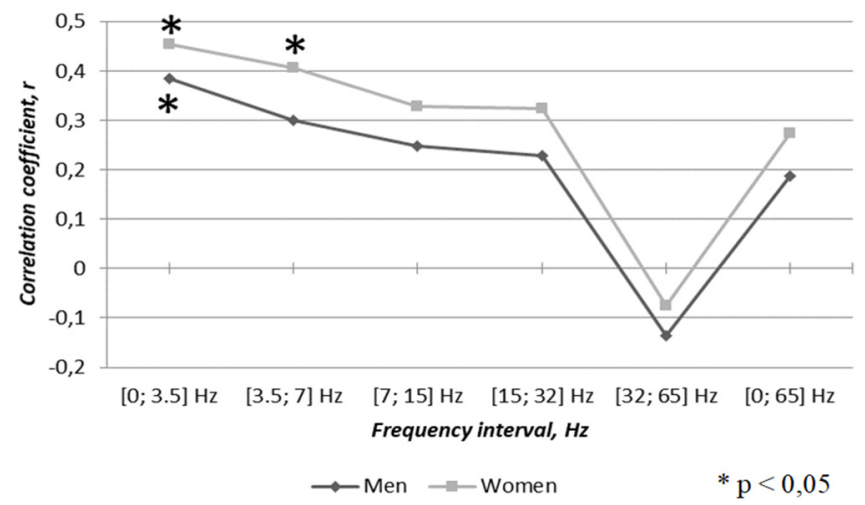

Fig. 4. Correlations between gender related cases of atrial fibrillation and TVMF changes through the first half of the year 2016

While, the second half of the year was analysed, (Fig. 5) negative correlation coefficients in all frequency ranges, except the SGamma range in males, while in females, the correlations varied from weak positive in the SDelta range to weak negative in the rest of the ranges were found. None of the correlation coefficients in the second half of the year were significant.

As approximately $90 \%$ of our study population had a history of $\mathrm{AH}$, we decided to extract them as a sub-study. For that, analyses on AFib concomitant with AH correlations with TVMF were performed and results were surprising. In whole year analyses, opposite results compared with general study population were found. In contrary to correlations found in general population, rates of AFib onsets in $\mathrm{AH}$ patients were higher in high frequency range (SGamma) through the year (Fig. 6). That supports the belief of SGamma range adverse effect on human health, as it was proved in AMI and ACS patients [9, 10] After the year was divided into two periods, results have switched and analysis of the first half of the year (Fig. 7) highlighted tendencies of negative 
correlation coefficients, but they were not significant. Meanwhile, analyses of the second half of the year (Fig.8) have demonstrated significant moderate positive correlation coefficients in all ranges except SDelta in both genders. These results, suggest, that AH patients might be more prone to experience AFib paroxysm during periods of increased high frequency (SGamma) range activity, while in the general AFib population, this range was associated with lower number of AFib cases.

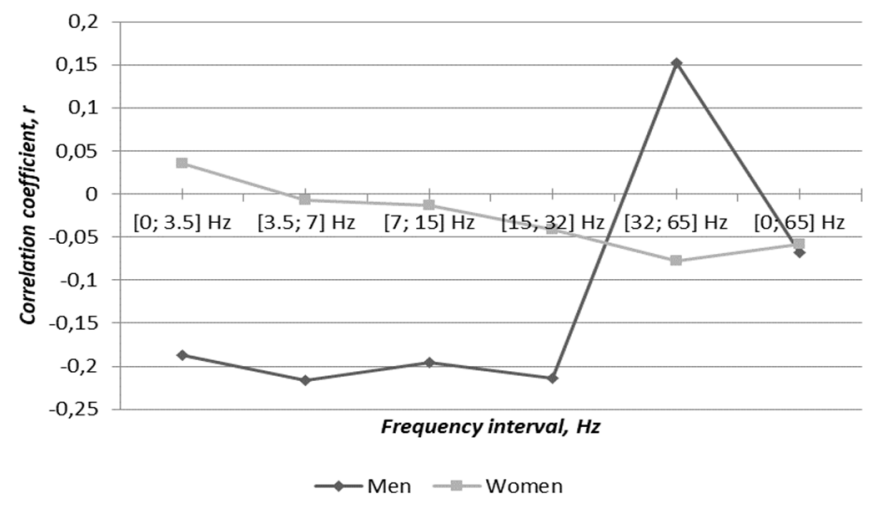

Fig. 5. Correlations between genders related cases of atrial fibrillation and TVMF changes through the second half of the year 2016

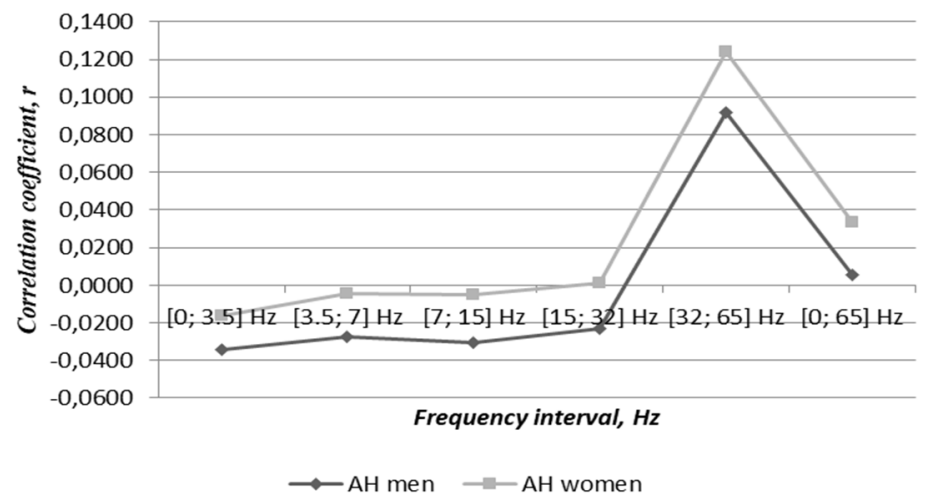

Fig. 6. Correlations between genders related cases of atrial fibrillation concomitant with arterial hypertension and TVMF changes through 2016

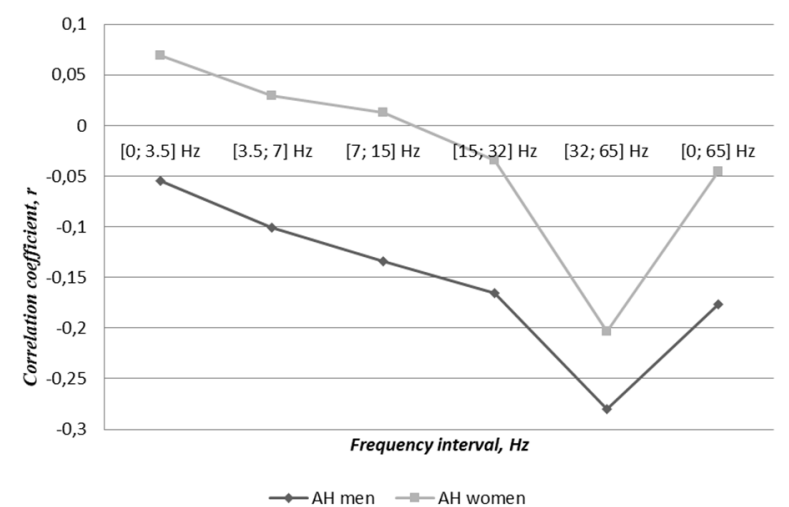

Fig. 7. Correlations between gender related cases of atrial fibrillation concomitant with arterial hypertension and TVMF changes through the first half of the year 


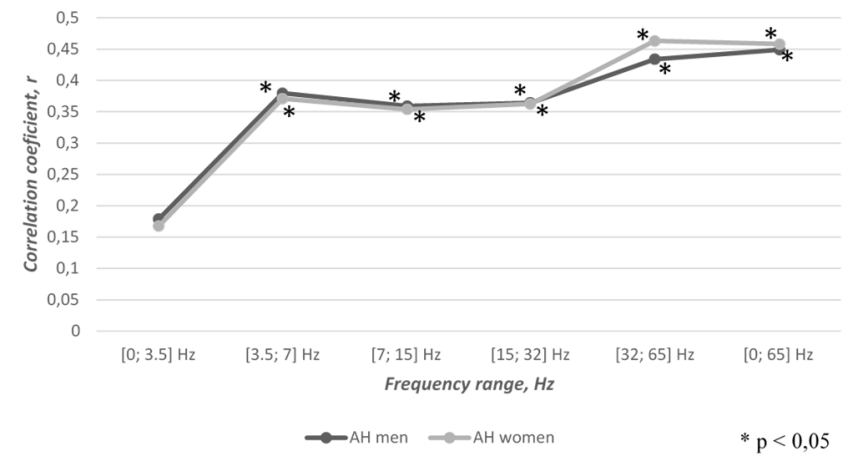

Fig. 8. Correlations between gender related cases of atrial fibrillation concomitant with arterial hypertension and TVMF changes through the second half of the year

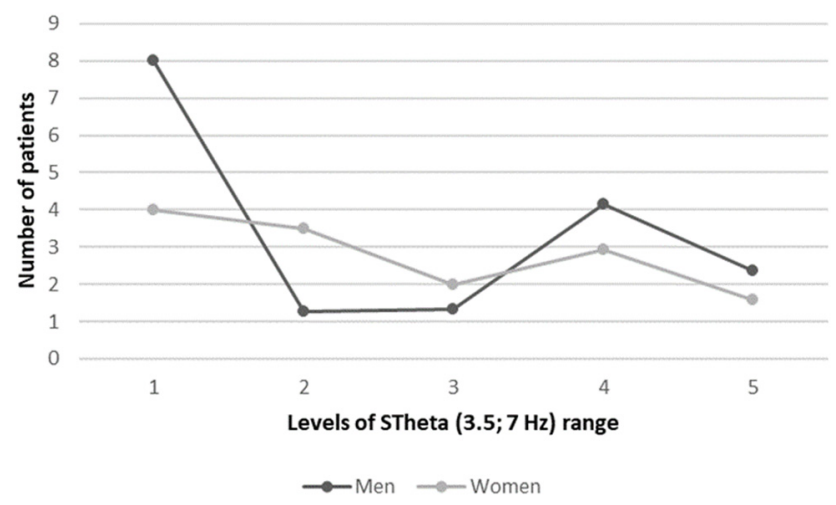

Fig. 9. Genders related cases of atrial fibrillation in five levels of TVMF power-varying magnetic field strength through the year

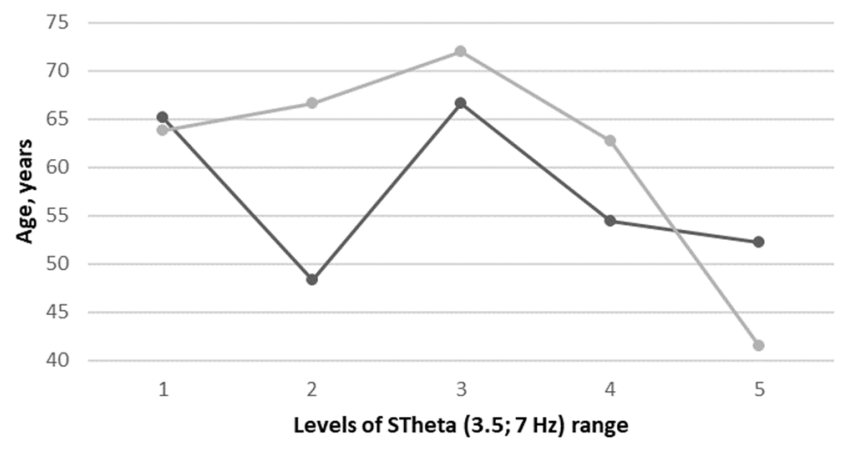

Fig. 10. Genders related atrial fibrillation patients' age distribution in five levels of TVMF power-varying magnetic field strength through the year

The final aspect of this research was to help understand more accurately relationship between AFib onsets and TVMF strength based on the differentiated entire TVMF range divided into 5 levels according to the strength. This part of analyses allowed us to define the most AFib causative strength intervals of the whole TVMF spectrum. The tendency of the lowest strength in low frequency range was found as the most AFib causative factor in general population, as the highest numbers of cases were admitted within the lowest level of TVMF strength in both genders (Fig. 9). Even more, correlation between age and AFib in accordance with TVMF strength levels during primary analyses was observed. The hypothesis that younger females may be more sensitive to 
the highest strength level of each range was confirmed. While younger males seem to be more sensitive to middle level of TVMF strength. As an example, correlations in levels of STheta range is given in Fig. 10. Results derived from these analyses were not significant but highlights promising tendencies and will be the subject for our further studies after inclusion of higher number of participants.

\section{Discussion}

Many new aspects of pathogenesis and causative factors for AFib including genetic predominance and atrial electrical remodelling have been recently proposed [3]. Numerous studies on environment parameters effect on human health with interesting and surprising results were published $[2,3,11-15,17,19-21,26,30]$. The majority of them analysed environment status relationship with acute coronary syndrome (ACS) $[11,12,17,22,27]$ or AH [17]. To our knowledge, this study is one in a few in which environment parameters' impact on AFib admissions was observed and the first analysing correlations between AAFib admission and TVMF changes.

Significant differences between the Global and the local Earth magnetic field on human regulatory systems have been widely recognised. Sometimes, changes in the Global Earth magnetic field (GMF) may not cause as noticeable local expression as would be expected. TVMF strength in different spots on the Globe at the same time significantly differs. The nearer to the polar cap, the stronger GMF influence on the changes in TVMF and vice versa nearer to the equator [30]. Not surprisingly, Patel et al. have reported some regions of the Globe as possibly provocative factor for some health disorders. They found higher prevalence of stroke, AH, diabetes mellitus (DM) and heart failure (HF) in Southern region of United States compared with the rest of the country [13]. Interactions between TVMF activity and human nervous system could be illustrated by overlaps between human brain activity waves and Schumann resonances, the resonating geomagnetic frequencies [14] (Table 1.). Due to that, as one of the most common mechanism of human cells regulatory systems and TVMF fluctuations interactions is explained through TVMF effect on human neural system including heart conducive system [2]. It was proposed to use cardiac rhythm variability parameters for overall evaluation of individual sensitivity to TVMF [14]. In consistence with Alabdulgande et al. [14] significant moderate correlations between AFib and changes in TVMF found in this study were not surprising.

New aspect of geomagnetism influence on cardiac rhythm regulation was that increased TVMF activity in low frequency ranges have provocative effect on cardiac arrhythmias, while otherwise adverse frequency range (SGamma, 32-65 Hz) seemed as favourable for patients with propensity for AFib. This, in contrary to general comprehension of TVMF influence on human heath, raises uncertainty whether SGamma range is really the only stressor frequency range, as SDelta and STheta ranges were found as AFib provocative factor in current study. This might be explained trough metabolic acceleration peculiarity of low frequency TVMF ranges which probably induce AFib [3]. Throughout those metabolic changes, onsets of AFib in patients with amyloidosis might be explained. There is strong evidence that the most frequent expression of heart involvement in systemic amyloidosis is AFib onsets [28]. There are several interesting studies showing amyloid deposits association with AFib. Surprisingly, AFib causing amyloids might be found only in atriums without any specific echocardiographic or systemic signs of amyloidosis [29]. That might be the answer, why in some cases AFib cannot be explained by basic pathogenesis of electrolytes disbalances or thyroid dysfunction. Even more, AFib onsets association with low frequency TVMF ranges, which are recognized as accelerating metabolism, might be explained by increased amyloid production and accumulation in heart tissues and according our data $\mathrm{AH}$ increase, accelerate this effect.

One more interesting attribute of this study is that the highest AFib admission peaks is repeated once in every 4 weeks (Fig. 2). These cyclical recurrences possibly repeat some cycles in environment. The duration of which most possibly correspond to lunar phases. It would not be 
surprising, as there are published several interesting documents on lunar activity influence on human circadian rhythm regulation [27, 30, 31] This might be illustrated by the case of a blind man, who was diagnosed with sleep-awake disorder and whose circadian rhythm was based on the motion of the moon rather than the sun $[11,27]$. Even more, Ooman et al. found significant differences between hospital admissions due to ACS on new moon versus full moon phases $[11,31]$. Interestingly, the new moon was found to be accountable for more ACS cases in contrary to general belief that full moon may cause health issues [11, 31, 32]. To be clear, not all health problems might be related with lunar phase as occurrences of cardiopulmonary resuscitations did not prove to be associated with lunar activity. We did not analyse possible interactions of lunar phase and AFib occurrence, but this could be a substrate for further studies.

Compelling results were driven from analyses of sub-study in which AFib concordant with $\mathrm{AH}$ were analysed. As $\mathrm{AH}$ is acknowledged risk factor (RF) for AFib [3, 6], we performed sub-analyses and impressive results were achieved. Numerous studies accredit AH as the most important RF for AFib in addition to other widely accepted including obesity, CD, HF $[2,3,5,6,9,10]$. Comorbidity of these two evidently strongly suggests a disbalance in human regulatory systems, which under exposure of TVMF fluctuations become even more disbalanced. Then compensatory mechanism become unable to restore homeostasis and the disease exacerbate $[2,6]$. Even more, TVMF effect on occurrence of isolated AH have been found [2]. Our analyses based on that evidence have shown great results. Stronger TVMF in low frequency ranges (SDelta, 0-3.5 HZ and STheta, 3.5-7 Hz) in concordance with AFib and AH is a significant factor for higher hospital admission rate due to AFib. To give more reliable evidence wider study population is necessary.

\section{Conclusions}

Significant correlation between acute atrial fibrillation and the local time varying Earth magnetic field changes was revealed.

Higher number of acute atrial fibrillation cases per week were associated with the high frequency range in males and with low frequency range in females.

Stronger low frequency magnetic fields appear to provoke atrial fibrillation in the general population in both males and females, while the high frequency range is associated with reduced atrial fibrillation admissions.

Atrial fibrillation concomitant with arterial hypertension during periods of increased high frequency range appears to have adverse effects on cardiac arrhythmias.

Changes in time varying magnetic field strength remarkably differs through the first and the second half of the year

Stronger magnetic fields in the lower frequency ranges through the year was associated with reduced admissions due to atrial fibrillation in arteria hypertension patients.

Stronger magnetic fields in higher frequency ranges through the second half of the year is associated with increases in admissions due to atrial fibrillation in arteria hypertension patients, while the first half of the year show a possible positive effect under stronger time varying magnetic fields in higher frequency ranges.

Lowest strength level of the low frequency range appears to be the most provocative factor in atrial fibrillation in the general population,

Young females were more sensitive to the highest strength level of each range, while young males showed highest sensitivity to the middle level of low frequency ranges.

\section{Limitations}

As this study was planned in equally to our previous study, it has similar limitations [11]. Firstly, there were included only patients admitted to one hospital omitting ones admitted into other hospitals in Kaunas and in other regions [11]. Secondly, the study population is low in 
number because of that we were not able to analyse patients more homogeneous groups. Thirdly, concomitant illnesses and biochemical status were not taking into consideration, but they might be affected by TVMF changes as well and may be culprit of AFib [11]. Further studies with higher study population number and counting concomitant illnesses and biochemical blood parameters are necessary [11].

\section{Acknowledgements}

Gratitude to the Head of the Cardiology department of the Hospital of Lithuanian University of Health Sciences Kaunas clinics for approval for the study implementation. Especial thanks we express for HMI personal, administration for possibility to make interesting investigations with Earth magnetic field.

\section{References}

[1] Camm A. J., Kirchhof P., Lip G. Y. H., Schotten U., Savelieva I., Ernst S., et al. Guidelines for the management of atrial fibrillation: the task force for the management of atrial fibrillation of the European Society of Cardiology (ESC). Europace, Vol. 12, 2010, p. 1360-1420.

[2] Chugh S. S., Havmoeller R., Narayanan K., Singh D., Rienstra M., Benjamin E. J., et al. Worldwide epidemiology of atrial fibrillation: a global burden of disease 2010 study. Circulation, Vol. 129, 2014, p. 837-847.

[3] Otway R., Vandenberg I., Guo G., Varghese A., Castro L., Liu J., et al. Stretch-sensitive KCNQ1 mutation. A link between genetic and environmental factors in the pathogenesis of atrial fibrillation? Journal of the American College of Cardiology, Vol. 49, 2007, p. 578-586.

[4] Chen Scarabelli C., Scarabelli T. M., Ellenbogen K. A., Halperin J. L. Device-detected atrial fibrillation. Journal of the American College of Cardiology, Vol. 65, 2015, p. 281-294.

[5] Passman R., Bernstein R. A. New appraisal of atrial fibrillation burden and stroke prevention. Stroke, Vol. 47, 2016, p. 570-576.

[6] Patel N. J., Deshmukh A., Pant S., Singh V., Patel N., Arora S., et al. Contemporary trends of hospitalization for atrial fibrillation in the united stated, 200 trough 2010 implications for healthcare planning. Circulation, Vol. 129, 2014, p. 2371-2379.

[7] Wake E., Brack K. Characterization of the intrinsic cardiac nervous system. Autonomic Neuroswcience: Basic and Clinical, Vol. 199, 2016, p. 3-16.

[8] Markides V., Schilling R. J. Atrial fibrillation: classification, pathophysiology, mechanisms and drug treatment. Heart, Vol. 89, 2003, p. 939-943.

[9] Yu L., Dyer J. W., Scherlag B. J., Stavrakis S., Sha Y., Sheng X., et al. The use of low-level electromagnetic field to suppress atrial fibrillation. Heart Rhythm, Vol. 12, 2015, p. 809-817.

[10] Selmer C., Olesen J. B., Hansen M. L., Lindhardsen J., Oslen A. M. S., Madsen J. C., et al. The spectrum of thyroid disease and risk of new onset atrial fibrillation: a large population cohort study. BMJ, 2012, p. 345.

[11] Žiubrytė G., Jaruševičius G., Landauskas M., McCraty R., Vainoras A. The local earth magnetic field changes impact on weekly hospitalization due to unstable angina pectoris. Journal of Complexity in Health Sciences, Vol. 1, 2018, p. 16-25.

[12] Jaruševičius G., Rugelis T., McCraty R., Landauskas M., Berškienė K., Vainoras A. Correlation between changes in local Earth's magnetic field and cases of acute myocardial infarction. International Journal of Environmental Research and Public Health, Vol. 15, 2018, p. 399.

[13] Liboff A. R. A role for the geomagnetic field in cell regulation. Electromagnetic Biology and Medicine, Vol. 29, 2010, p. 105-112.

[14] Alabdulgader A., Mccraty R., Atkinson M., Vainoras A., Berškienė K., Mauricienė V., Daunoravičienė A., Navickas Z., Šmidtaitė R., Landauskas M. Human heart rhythm sensitivity to earth local magnetic field fluctuations. Journal of Vibroengineering, Vol. 17, 2015, p. 3271-3278.

[15] Gaetani R., Ledda M., Barile L., Chimenti I., De Carlo F., Forte E., Ionta V., Giuliani L., D'emilia E., Frati G. Differentiation of human adult cardiac stem cells exposed to extremely low-frequency electromagnetic fields. Cardiovascular Research, Vol. 82, 2009, p. 411-420.

[16] Electromagnetic Hypersensitivity. International Workshop on Electromagnetic Field Hypersensitivity, World Health Organization, 2006. 
[17] Gmitrov J., Gmitrova A. Geomagnetic field effect on cardiovascular regulation. Bioelectromagnetics, Vol. 25, 2004, p. 92-101.

[18] Breus T., Komarov F., Musin M., Naborov I., Rapaport S. Heliophysical factors and their influence on cyclical processes in biosphere. Itogi Nauki I techniki: Medicin. Geogaf, Vol. 18, 1989, p. 172-174.

[19] Palmer S. J., Rycroft M. J., Cermack M. Solar and geomagnetic activity, expremely low frequency magnetic and electric fields and human health at the Earth's surface. Surveys in Geophysics, Vol. 27, 2006, p. 557-595.

[20] Mavromichalaki H., Preka-Papadema P., Theodoropoulou A., Paouris E., Apostolou Th. A study of the possible relation of the cardiac arrhythmias' occurrence to the polarity reversal of the solar magnetic field. Advances in Space Research, Vol. 59, 2017, p. 366-378.

[21] Cornellisen G., Halberg F., Breus T., Syutkina E., Baevsky R., Weydahl A., Watanabe Y., Otsuka K., Siegova J., Fiser B., Bakken E. F. Non-photic solar associations of heart rate variability. Journal of Atmospheric and Solar-Terrestrial Physics, Vol. 64, 2002, p. 707-720.

[22] Stoupel E., Domarkiene S., Radisauskas R., Bernotiene G., Abramson E., Israelevich P., Sulke J., Breus T. Variants of acute myocardial infarction in relation to cosmophysical parameters. Seminars in Cardiology, Vol. 11, 2005, p. 51-55.

[23] Hardell L. World Health Organization, radiofrequency radiation and health - a hard nut to crack (Review). International Journal of Oncology, Vol. 51, 2017, p. 405-413.

[24] Pall M. L. Microwave frequency electromagnetic fields (EMFs) produce widespread neuropsychiatric effects including depression. Journal of Chemical Neuroanatomy, Vol. 75, 2016, p. 43-51.

[25] Huss A., Peters S., Vermeulen R. Occupational exposure to extremely low-frequency magnetic fields and the risk of ALS: a systematic review and meta-analysis. Bioelectromagnetics, Vol. 39, 2018, p. 156-163.

[26] Landauskas M., Vainoras A., Ragulskis M. Algebraic and spectral analysis of local magnetic field intensity. Proceedings of the Lithuanian Mathematical Society, Ser A, Vol. 56, 2015, p. 54-59.

[27] Miles L. E., Raynal D. M., Wilson M. A. Blind man living in normal society has circadian rhythms of 24.9 hours. Science, Vol. 198, 1977, p. 421-423.

[28] Kabach M., Zaiem F., Alraies M. C. Cardiac amyloidosis presenting with paroxysmal atrial fibrillation. QJM: An International Journal of Medicine, Vol. 108, 2015, p. 819-820.

[29] Timofejeva I., McCraty R., Atkinson M., Joffe R., Vainoras A., Alabdulgader A. A., Ragulskis M. Identification of a group's physiological synchronization with earth's magnetic field. International Journal of Environmental Research and Public Health, Vol. 14, 2017, p. 998.

[30] Sukhacheva T. V., Eremeeva M. V., Ibragimova A. G., Vaskovskii A. V., Serov R. A., Revishvili A. Sh. Isolated atrial amyloidosis in patients with various types of atrial fibrillation. Bulletin of Experimental Biology and Medicine, Vol. 160, 2016, p. 844-849.

[31] Oomman A., Ramachandran P., Shanmugapriya, et al. A novel trigger for acute coronary syndromes: the effect of lunar cycles on the incidence and in-hospital prognosis of acute coronary syndromes - a 3-year retrospective study. Journal of the Indian Medical Association, Vol. 101, 2003, p. 227-228.

[32] Alves D. W., Allegra J. R., Cochrane D. G., Cable G. Effect of lunar cycle on temporal variation in cardiopulmonary arrest in seven emergency departments during 11 years. European Journal of Emergency Medicine, Vol. 10, Issue 3, 2003, p. 225-228. 